\title{
eGovGrid: A Service-Grid-Based Framework for E-Government Interoperability
}

\author{
Dongju Yang, Yanbo Han, and Jinhua Xiong \\ Research Centre for Grid and Service Computing, \\ Institute of Computing Technology, Chinese Academy of Sciences, \\ 100080 Beijing, China \\ \{yangdongju, yhan, xjh\}@ict.ac.cn \\ WWW home page: http://vega.ict.ac.cn
}

\begin{abstract}
Interoperability has been regarded as a major issue in implementing e-Government systems. In this paper, a service grid based framework for the interoperability - eGovGrid, is proposed, which facilitates "horizontal" resource sharing and interoperability among "vertical" e-Government subsystems. Key concepts and technologies include an ontology-based eGovernment service metadata model, a service repository serving as a virtual pool of heterogeneous e-Government services, and corresponding architecture. The effectiveness of eGovGrid in constructing e-Government systems is demonstrated with a real case.
\end{abstract}

\section{Introduction}

Resource integration and interoperation among different government departments are of key importance for today's e-Government systems, in which information systems from different departments need to be integrated. There are two factors that hinder direct interconnection and interoperation. One is resource heterogeneity with diversity in resource formats, runtime environments, programming languages, interface contracts, etc. The other is semantic ambiguity, which results in misunderstanding among different information systems. Therefore, an e-government framework must be based on an open architecture, encapsulating heterogeneous resources with unified interface, logically centralizing and unified managing these resources, supporting interoperability based on semantic infrastructure, and let endusers use dynamic resources in secure, transparent, flexible and uniform environments. 
There are two kinds of approaches addressing e-government interoperability: one is based on semantic web or semantic driven $[2,3,4,5]$, another one is based on crossorganizational workflow $[6,7,8,9]$. But most of the above works just provide basis or general ideas for the development of e-Government system, and are lack of operational instructions, a solid framework, related data models or software infrastructure to address the problems in an e-Government environment.

Service grid [11], a kind of combination of grid computing [10] and SOA technologies, helps to open up a new way for cross-organizational resources integrating and collaboration in e-government. Service grid technologies can be used to build the platform for resource sharing in e-government systems, and also bring new features of better reusability, flexibility and scalability.

Consequently we proposed a service-grid-based framework for e-government interoperability, named eGovGrid, which targets at facilitating resource sharing among "horizontal" organizations and interoperability among "vertical" eGovernment subsystems. Hereinafter, "horizontal" means cross-organizational application, and "vertical" means information system within one organization. In eGovGrid, an ontology-based service metadata model is proposed. A service repository serving as a virtual pool of distributed e-Government services takes this model as basis. The key technologies are implemented and demonstrated in an egovernment application of a city in southern China. As a result, seven departments, two counties and five companies have already been interconnected; then resource sharing and exchange are enabled. The preliminary result illustrates that: the eGovGrid framework enables interoperability among the above organizations; heterogeneous resources can be integrated dynamically and effectively.

\section{Architecture of eGovGrid}

The traditional pattern of "information center" adopts centralized data storage, which can not easily adapt to an open e-government environment due to non real-time update and high cost for management and maintenance of centralized data. Web services provide a loosely-coupled mechanism to encapsulate resources with standardized interfaces, which enables users to plug in/out resources dynamically. Users only need to use Web services, don't need to concern, manage, and maintain resource objects behind the Web services. However, there are several challenges needed to be addressed when using Web services. First, WSDL is used to describe implemental interface of Web services without semantic, which results in that the services are hard understood by end users, and interoperation between services are not easy to be achieved. So an end-user-understandable service metadata model is required. Second, UDDI severs as a discovery service for the WSDL descriptions. It can not work well with changing models.

Motivated by the above challenges, we proposed "business service" as an abstraction of Web services at business level. An ontology-based service metadata model is proposed to describe business services to achieve user-understandability and interoperability, and service repository serves as a virtual resource pool to enable resources physically distributed storage and logically centralized management. The 
service repository is responsible for creation, organization, and management of business services, and provides a unified service directory for service browsing and search. A service runtime environment is also provided for services discovery and invoking. The architecture of e-government applications is illustrated in Fig. 1.

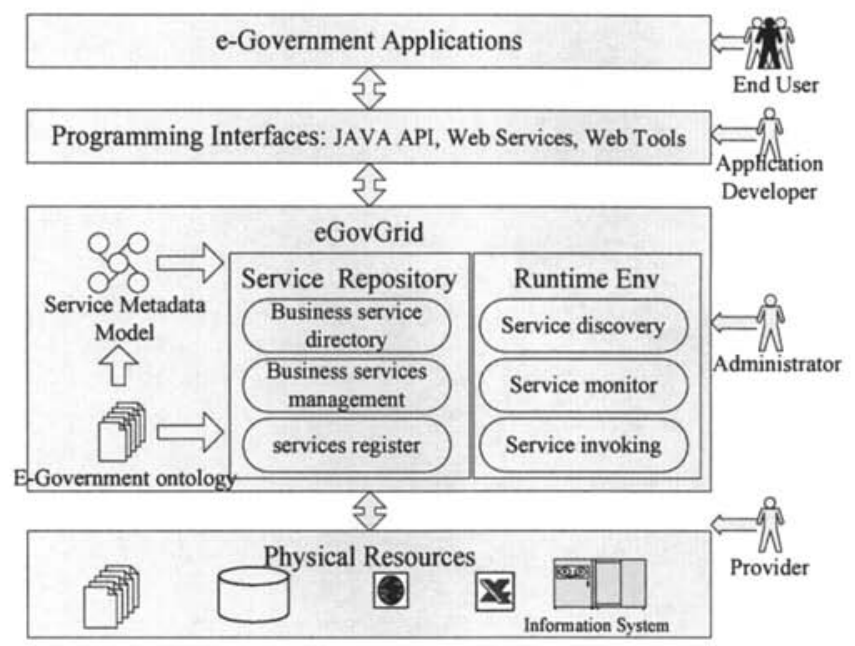

Fig. 1. Architecture of eGovGrid

\subsection{Business service and its metadata model}

Business service is the business-level abstraction of Web services to achieve usercentric understandability and interoperability. Business service needs to be browsed and searched by end users, support interoperability between applications, and be executed to access the behind physical resources. It is necessary to monitor services status and analyze their usage and quality to provide statistical data to users. Furthermore, appropriate authority is necessary in order to prevent misuse of services. And an extensible metadata model is helpful to build flexible and adaptable application.

OWL-S [12] and WSDL-S [13] are two notable service description languages to describe semantic Web services. However, OWL-S aims at automatic Web service composition and interoperation by describing the prerequisites and consequences of application of individual services, which makes it too complex to use; WSDL-S is an extension of WSDL, and its emphasis is still to describe implementation interface of services. So these two languages are not suitable for business service description. Another wide-used resource metadata model is Dublin Core Metadata Element Set [14], which is a set of vocabulary of fifteen properties used for resource property 
description. But DC focuses only on for information resources, such as a book in digital library, and not suitable for business services.

Motivated by the above requirements our service metadata model takes EGovernment ontology as basis and comprises six facets, illustrated in Fig. 2. Hereinafter, e-Government ontology is described by OWL [1] and aims at normatively describing basic concepts, relationships between objects, and interfaces in e-Government application. It can provide the vocabulary base for referring to the terms in certain subject areas, and the logical statements [15]. E-Government ontology can define the metadata elements (leaf nodes in the model), define the value range of one metadata elements, and describe the relationships of metadata elements, so as to facilitate the metadata model more clear and accurate without misunderstanding, and support model evolution based on ontology reasoning.

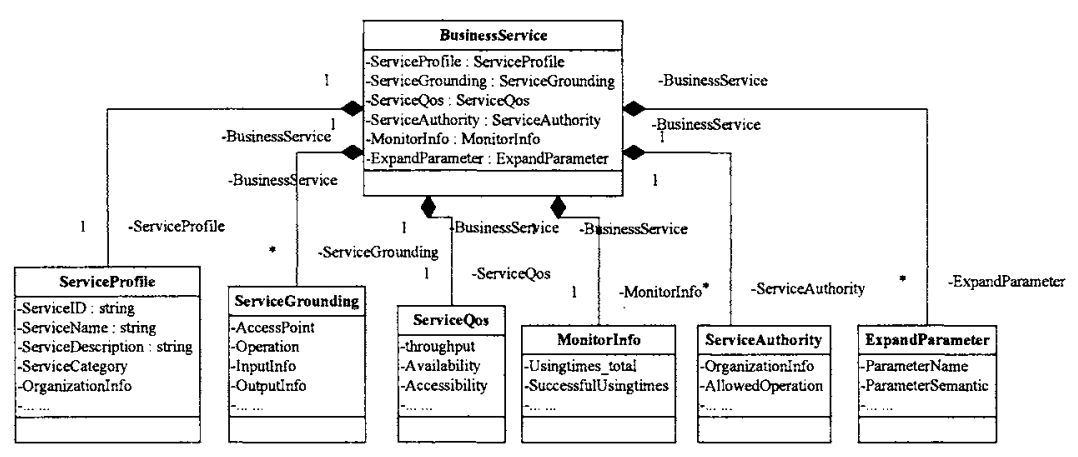

Fig. 2. Service metadata model

- ServiceProfile is to describe the basic properties of a service, such as service name, description, organization info (provider), service category, etc. which helps end users to know what it is.

- ServiceGrounding is to describe the implementation properties of a service, such as access point, operation, input, and output. The latter three items must be specified with semantic annotation to identify their relationships, so as to enable interconnection and interoperation of services. A BusinessService may have more than one ServiceGrounding, which indicates that many Web services can implement this business service. ServiceGrounding is used for service addressing and invoking.

- ServiceQos is to describe the QOS of a service, such as throughput, availability, accessibility, etc. which is used for service evaluation.

- MonitorInfo is to describe the usage of a service and give some statistical data, such as using times and successful using times within some periods, which is used for service evaluation.

- ServiceAuthority is to describe which organizations have authorities to use the service and which operations are allowed. Then manager of the 
organization can assign her/his authority to other users within organizations. So an authority chain is produced. ServiceAuthority is the important properties to prevent misuse of services.

- ExpandParameter is to describe the additional properties of services, which may be required in some contexts, such as price, available time, etc. ExpandParameter is used to achieve extensibility and adaptability.

The values of the above metadata elements may be filled out in three ways. The first is that providers describe some service properties during registration, such as ServiceProfile, ServiceQos, and ServiceAuthority. The second is that the information system automatically parses the WSDL file to fill out some properties, such as ServiceGrounding. The last one is that service runtime environment provides and modifies some properties by analyzing the usage of services, such as MonitorInfo and ServiceQos.

Compared with other existing service metadata model $[12,13,16]$, the major contribution is that our service metadata model includes not only static but also dynamic properties, such as service usage and service quality. Authority property is also brought into model. All of these properties ensure integrity of the services information and make services use and management easy.

\subsection{Service repository}

Service repository aims at building a centralized services pool, to enable registration, unified management, and discovery of business services. It brings out a lot of challenges when adopting service repository to build application.

First, a service repository is taken as the basis to build various applications with different requirements. One unified metadata model may be not enough to describe all kinds of services clearly, so multiple service metadata models in one repository are necessary. We take the previous service metadata model as core metadata model. Each metadata model in service repository is an extension of core metadata model. Therefore, our service repository can satisfy requirements of different applications with extension mechanism, and enable interoperability among different services with core metadata and its ontology basis.

Second, when registering a service into the service repository, the provider should select the appropriate service metadata model and fill out each metadata element. It is a challenging task for the provider to associate proper semantic annotation to the value. How to quickly locate the proper ontology classes or properties from semantic database is the major issue. Our approach is to automatically recommend the proper ontology classes and properties according to the context based on initial recommendation mechanism.

Third, the effectively organizing and managing services are necessary to facilitate service browsing, querying and discovery. In service repository, an ontology-based taxonomy is built, so that services can be aligned with trees with many roots according to their categories and relationships defined in ontology base.

Finally, an end-user friendly tool is required for services browsing, querying and invoking. A service directory supports users to browse services from different views. Furthermore, service query language enables flexible query of services, which is 
similar to SQL in database. A runtime engine enables service parsing and addressing, and then submits the invoking requirements to runtime environment, and acquires the result in synchronous or asynchronous way.

Based on the service repository, cross-organizational e-government applications can be constructed to enable unified management of services, without concerning heterogeneous and distributed resources behind of services. The service repository also supports direct access to business services, which will be dynamically bound (interpret) to corresponding physical resources.

\section{Applications}

In recent years Chinese government are experiencing the transformation from management-oriented government to service-oriented government. In order to provide more convenient services to citizens and companies, government must effectively organize internal and external resources on-demand. eGovGrid took the real requirements from a southern China city, and has already been partly demonstrated in an e-Government system project of the city. The goal of this project is to build a virtual resource center to enable integration of all resources distributed in multiple departments and transparent access to these resources in a unified way.

Aiming at the above goal, a resources interoperation platform with virtual resource center is implemented. Seven departments, five companies, and two counties are taken as demonstration units to exchange economic information via this platform.

First, by analyzing business requirements, existed or new e-Government ontology is imported or created to build semantic infrastructure. There are two kinds of services related to economic information exchange. One is information retrieving service, which encapsulates information resources for retrieving, and another one is information transaction service, which encapsulates information system for more operations, such as updating, analyzing, etc. Accordingly, two kinds of service metadata models are designed. One is for information retrieving service and emphasizes on description of on-behind information resources; another is for information transaction service and emphasizes on description of service operation.

Second, over thirty information retrieving services and information transaction services are registered into the virtual resource center. The description contents are based on the above two service models.

Based on service directory, users can browse services in multiple views, or query services by service query language. They can submit their request for service invoking, so as to transparently access to physical resources.

Interoperability is easily achieved when constructing virtual resource center with eGovGrid. The following is a comparison of the processes of exchange information with and without eGovGrid support (Fig. 3). Without eGovGrid, the process could be divided into several steps: an end user brings forward requirements; a provider exports data from the original information system; the provider sends data to the end user; the end user checks the data; the end user imports the data to the target information system. If there are multiple exchange requirements, the process needs 
to be repeated multiple times. Now with eGovGrid support, the exchange information process is divided into two stages: "preparation state" and "exchange stage". In "preparation stage", end users and providers share their read and write interface of information system. When information needs to be exchanged, end user retrieves and connects two services (read interface from other system and write interface to own system) to form a process, and then invokes the process to finish information exchanging. If there are multiple exchange requirements only the second stage needs to be repeated.

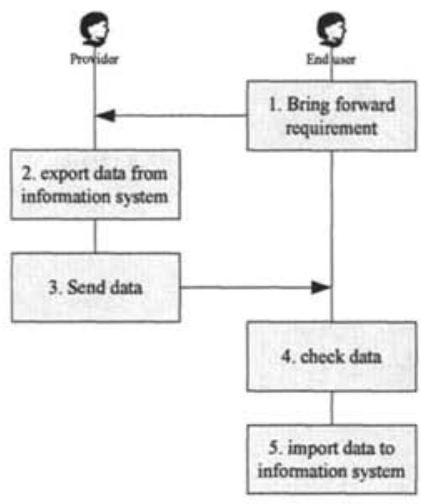

One process of exchange information without eGovGrid

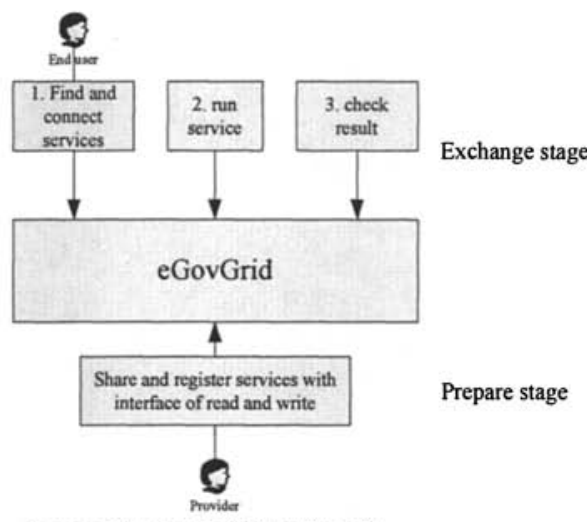

Process of exchange information with eGovGrid

Fig. 3. Comparison of exchange information process, without and with eGovGrid support

Furthermore, resources are logically centralized management without changing physical storage and the maintenance is undertaken by each provider. Resource can be plug in/out dynamically. Extensibility is also achieved, including organization extension, user extension, services extension, service metadata model extension etc.

\section{Conclusions}

eGovGrid proposed in the paper is a useful trial. It facilitates "horizontal" resource sharing and interoperability among "vertical" e-Government subsystems. Key concepts and technologies include ontology-based e-Government service metadata model, a service repository, i.e. a virtual pool of heterogeneous e-Government services, and corresponding architecture. The effectiveness of eGovGrid in constructing e-Government systems is demonstrated with a real case in a southern China city. 
Many important issues are still work-in-progress, which include how to deal with various loads of concurrent requests for service at peak times, how to deal with application change, how to build the appropriate ontology at runtime, how to deal with authority and security, etc.

\section{Acknowledge}

This paper is supported by the National Natural Science Foundation of China under Grant No. 70673098, National Basic Research Program of China (973 Program) under Grant No. 2007CB310805 and Open Funding of SKLSE of China under Grant No. SKLSE05-08.

\section{References}

1. OWL Web Ontology Language Guide, Michael K. Smith, Chris Welty, and Deborah L. McGuinness, Editors, W3C Recommendation, 10 February 2004, http://www.w3.org/TR/2004/REC-owl-guide-20040210/ . Latest version available at http://www.w3.org/TR/owl-guide/.

2. R. Klischewski. Semantic Web for e-Government. Proceedings of EGOV 2003.

3. Luis 'Alvarez Sabucedo and Luis Anido Rif'on, A Proposal for a Semantic-Driven eGovernment Service Architecture, EGOV 2005, LNCS 3591, pp. 237-248, 2005.

4. Witold Staniszkis, Edyta Kałka, Grzegorz Nittner, Eliza Staniszkis, and Jakub Strychowski, Integration of Pre-existing Heterogeneous Information Sources in a Knowledge Management System, EGOV 2004, LNCS 3183, pp. 507-514, 2004.

5. Matti Järvenpää, Maiju Virtanen, Airi Salminen, Semantic Portal for Legislative Information., EGOV 2006, LNCS 4084, pp. 219-230, 2006

6. D. Gouscos, G. Mentzas \& P. Georgiadis, PASSPORT : A Novel Architectural Model for the Provision of Seamless Cross-Border e-Government Services, 12th International Conference on Database and Expert Systems Applications (DEXA 2001), Munich, September 2001, IEEE Computer Society Press, pp. 318-322.

7. Giannis Verginadis, Dimitris Gouscos, Maria Legal, Gregoris Mentzas, An Architecture for Integrating. Heterogeneous Administrative Services. into One-Stop e-Government, the eChallenges 2003 Conference, Bologna.

8. Euripidis Loukis and Spyros Kokolakis, Computer Supported Collaboration in citizens Sector: The ICTE-PAN Project, EGOV 2003, LNCS 2739, pp. 181-186, 2003.

9. D. Gouscos, G. Laskaridis, D. Lioulias, G. Mentzas \& P. Georgiadis, An Approach to Offering One-Stop e-Government Services: Available Technologies and Architectural Issues, e-Government: State of the Art and Perspectives Conference (EGOV 2002), Aix-en-Provence, September 2002.

10. Foster, I., C. Kesselman, and S. Tuecke, The Anatomy of the Grid: Enabling Scalable Virtual Departments. International J. Supercomputer Applications, 2001. 15(3)

11. Weissman, J.B. and B.-D. Lee, The Service Grid: Supporting Scalable Heterogeneous Services in Wide-Area Networks. In Proc. of Symp. Applications and the Internet, San Diego, CA, 2001: p. 95-104. 
12. Coalition, O.S.: Owl-s: Semantic markup for Web services. Web available (2005) http://www.daml.org/services/owl-s/l.1/.

13. Rama Akkiraju, Joel Farrell, John Miller, Meenakshi Nagarajan, Marc-Thomas Schmidt, Amit Sheth, Kunal Verma, Web service Semantics - WSDL-S, http://www.w3.org/Submission/WSDL-S/

14. Dublin Core Metadata Element Set, Version 1.1, http://dublincore.org/documents/dces/ 15. Antoniou G, Harmelen F. A semantic web primer[M]. The MIT Press, 2004.

16. Broekstra, J., Ehrig, M., Haase, P., van Harmelen, F., Kampman, A., Sabou, M., Siebes, R., Staab, S., Stuckenschmidt, H., and Tempich, C. A metadata model for semantics-based peerto-peer systems. In Proceedings of the 1st Workshop on Semantics in Peer-to-Peer and Grid Computing at the 12th International World Wide Web Conference. Budapest, Hungary. 2003. 